\title{
Deserción Universitaria: Nuevo Análisis Metodológico
}

\author{
Arturo B. Rodriguez ${ }^{(1)}$, Jaime Espinoza ${ }^{(1)}$, Leonardo J. Ramirez ${ }^{(2)}$ y Angelica Ganga ${ }^{(3)}$ \\ (1) Univ. de Santiago de Chile, Depto de Tecnología Industrial, Grupo de Investigación en Nuevas \\ Tecnologías (GINT), Santiago, Chile. (e-mail: arturo.rodriguez@usach.cl; jaime.espinoza@usach.cl) \\ (2) Universidad Militar de Nueva Granada, Facultad de Ingeniería, Grupo de Investigación TIGUM, Bogotá, \\ Colombia. (e-mail: leonardo.ramirez@unimilitar.edu.co) \\ (3) Universidad Santiago de Chile, Departamento de Ciencia y Tecnología de los Alimentos, Laboratorio de \\ Biotecnología y Microbiología Aplicada (LAMAP). Santiago-Chile. (e-mail: angelica.ganga@usach.cl)
}

Recibido Abr. 23, 2018; Aceptado Jun. 19, 2018; Versión final Jul. 25, 2018, Publicado Dic. 2018

\begin{abstract}
Resumen
El presente artículo propone un nuevo indicador para medir la deserción por departamento y por carrera de programas universitarios, denominado Índice Geométrico de Deserción. Además, se define un parámetro denominado permanencia, que mide el porcentaje que representa la cohorte en cada año de permanencia en el sistema. Los resultados indican que el departamento tiene una deserción en crecimiento sostenido, lo que preocupa a los directivos de la institución. Todas las carreras tienen una deserción que tiende al crecimiento excepto las carreras C1 y C3. Es necesario revisar los resultados y reflexionar sobre la tendencia al alza del IGD y analizar sus causas, para el desarrollo de políticas que logren revertir esta tendencia.
\end{abstract}

Palabras clave: deserción; educación; calidad de la enseñanza, retención, gestión educativa

\section{University Dropout: New Analysis methodology}

\begin{abstract}
This article proposes a new indicator to measure desertion from university programs classified by departments and by careers, indicator called Geometric Dropout Index. In addition, a parameter called permanence is defined, which measures the percentage that the cohort represents in each year of permanence in the system. The results indicate that the department has a steady growing dropout, which worries the directors of the institution. All programs have a dropout that tends to grow except for career $\mathrm{C} 1$ and C3. It is necessary to review the results and reflect on the upward trend of the IGD and analyze its causes for the development of policies that reverse this trend.
\end{abstract}

Keywords: dropout; education; quality of teaching, retention, education management 


\section{INTRODUCCIÓN}

En los últimos años los desarrollos de las instituciones educativas se centran en el mejoramiento de los indicadores, observables en los procesos de certificación, aunque el logro del indicador no significa necesariamente el mejoramiento de aquello que indica. Estos indicadores como tasa de titulación, retención de primer y segundo año, porcentaje de jornada completa equivalente, etc., son altamente discutidos y mostrados en diferentes estudios que nos permite ver que efectivamente algunas instituciones logran estándares aceptables y otras no. Sin embargo, los indicadores individualizados dicen poco respecto de lo que se busca garantizar en los procesos de certificación y aseguramiento de la calidad. La tasa de retención y titulación son quizás los indicadores más relevantes entre otros, existiendo diferentes formas de evaluarlos, por lo general se realiza estudios por cohorte donde se relaciona un grupo respecto del año anterior. En Chile, el Sistema de Información de la Educación Superior, SIES (2016), indica que la deserción para el año 2015 es 22,50\%, además en el periodo 2008-2102 es de un 24,7\% (Miranda et al., 2017). Estas cifras empeoran cuando revisamos los cursos e-Learning y b-Learning como los MOOC (Massive Open Online Courses) o cursos abiertos masivos online donde la deserción alcanza el $90 \%$ y $50 \%$ respectivamente (Grefori et al., 2018).

Mientras en Norteamérica la deserción disminuyó de 10,9 por ciento en el 2000 al 5,9 por ciento el 2015, lo que contrasta con las tasas en Chile que alcanzan el 22,5 por ciento. Además, en los últimos cinco años (2010-2015) la tasa disminuyó de 7,4 a 5.9 por ciento. En los últimos 15 años la gradiente de deserción masculina (12.0 a 6.3 por ciento) fue mayor a la femenina (9.9 a 5.4 por ciento), sin embargo, la tasa final de deserción es mayor para la masculina. También la tasa de deserción de la comunidad de blanca es menor a la comunidad negra, siendo ambas más bajas que la comunidad hispana. Es observable que la brecha entre jóvenes blancos, negros e hispanos se redujo a 1,9 y 4,6 puntos porcentuales respectivamente para el periodo 2000-2015. (McFarland et al., 2017). Es interesante la preocupación de los países por la deserción y la implementación de monitoreo de las comunidades desde una observación integradora. Otro indicador de importancia en la literatura internacional es tasa de graduación o titulación la misma que la OCDE estima como el porcentaje que una cohorte se gradúe durante el tiempo estipulado al programa. En los últimos años los reportes utilizan la nueva clasificación CINE 2011 que orienta la información estadística sobre los egresados de la educación terciaria (universitaria).

El año 2016 los estudiantes universitarios de países pertenecientes a la OCDE tenían un 49\% de probabilidad de graduarse inclusive los estudiantes extranjeros que estudian en estos países. Hay que precisar que estos indicadores se basan en egresados que obtienen el título por primera vez. De esta forma, siempre se observa que la deserción de primer año es siempre mayor que la del segundo año. Sin embargo, existen muchas excepciones como, por ejemplo, estudiantes que se trasladan de institución, de facultades de la misma institución, etc. que no son tomados en cuenta por los indicadores (Montes-Gutiérrez I. et al., 2010). Es evidente que la educación debe avanzar en mejorar los indicadores, para que dichos índices proyecten lo que verdaderamente pretenden medir, y sirvan de modelo predictor y controlador de las medidas realizadas para mejorar su disminución hasta el mínimo posible. También hacer observable que medidas hacen falta realizar para conseguir un mejor y correcto funcionamiento. La calidad de la educación no es un conjunto de indicadores que determinan si una institución es o no es de calidad. Un caso interesante de observar es el de (Alegre de La Rosa et al., 2017), que contrasta diferentes indicadores como tasa de éxito, eficiencia, graduación, deserción y rendimiento de varias carreras con la opinión de sus comisiones de calidad, los resultados arrojaron que solo las ciencias de la salud logran una mejora de la tasa de éxito en 3 años, esto contrasta con los resultados en Ingeniería y Arquitectura. Para poder entender la calidad es necesario ser capaces de establecer formas que revelen el verdadero funcionamiento del sistema, porque es la única forma de corregirlo, solo son alcanzables las metas que se basan en registros objetivos de la realidad. Se han establecido en la literatura diferentes formas y metodologías para determinar la deserción o dropout desde la deserción por cohorte hasta la deserción total.

Se han desarrollado estudios que tratan de modelarla a través de estilos de aprendizaje (Rodriguez et al. 2010), aspectos sociales, aspectos académicos, etc. La literatura indica que las razones de la deserción se encuentran dentro de la institución y no fuera de ella, por otro lado, existe consenso sobre que la deserción del primer año es superior al resto de los años de la duración de la carrera, también existe literatura que contrasta indicando que para permanecer con éxito en la universidad son necesarias habilidades transversales que los estudiantes deben tener antes del ingreso a la institución educativa (Baeza-Rivera et al., 2016), inclusive algunos mencionan que una elevada autoestima es de relevante importancia para el desarrollo dentro de la universidad (Suhlmann et al., 2018). Otros logran establecer la importancia de la vocación como elemento que permite la permanencia en el sistema educativo (Esteban et al., 2017). Por lo demás, hay estudios que indican que las razones económicas solo explican del 3 al $5 \%$ del total de la deserción. Los Indicadores de deserción y titulación se establecen por año de la carrera, pero no existe una metodología que permita establecer la deserción del programa, carrera o facultad y, de hecho, de la 
universidad; por lo general se utiliza el promedio aritmético de las deserciones anuales de las unidades individuales. La nueva propuesta permite una medición basada en información continua de las cohortes a través de los años y propone una medición geométrica, es decir basada en el cálculo del área respecto de la permanencia de deserción 0 , esto permite un cálculo de mayor precisión y contrasta con las mediciones propuestas en la literatura que se basan en promedios comparados con el inicio y final de un periodo. El presente estudio presenta una propuesta geométrica para el cálculo de la deserción el mismo que se ha denominado Índice Geométrico de la Deserción (IGD) el mismo que se procederá a su explicación y desarrollo.

\section{METODOLOGÍA}

Como se mencionó antes, es de suma importancia observar las reflexiones sobre las razones de la deserción; algunos autores mencionan, que estás se encuentran orientadas hacia lo que sucede después del ingreso y no a la precedencia (Grosset et al., 1991). Sin embargo, es necesario profundizar sobre la metodología de medición de este indicador. Solo así se logrará transitar por algoritmos convergentes hacia el logro que el "Aprendizaje Realmente Ocurra" (ARO), que sin lugar a duda es el objetivo supremo de la educación. Muchos investigadores han estudiado el problema, el modelo de Tinto que ha sido muy referenciado y, además, muestra la gran complejidad de medir la deserción (Tinto et al., 1989). muy congruente con los trabajos de (Durkeim et al., 1992; Spady et al., 1970), relacionando las conductas anteriores al ingreso, con los resultados posteriores al ingreso. Además, la relación existente entre el proceso de deserción y los vínculos afectivos de la gestión (Pascarella et al., 1980). Existen muchas definiciones de la deserción; por ejemplo, algunos definen la deserción como el alejamiento de su programa de estudios sin obtener el título o grado atingente al programa, tomando en cuenta un tiempo donde es inexistente la posibilidad de reincorporación, sin embargo además se propone la distinción entre la deserción voluntaria e involuntaria, donde se menciona que la deserción voluntaria puede estar presente cuando el estudiante renuncia o abandona sin comunicación a la institución, por otro lado la deserción involuntaria aparece como consecuencia de las decisiones de la instituciones basadas en su reglamentación y normatividad (Himmel, 2002).

El equipo de investigación no comparte esta clasificación debido a que la deserción en todo momento es responsabilidad de la institución, la clasificación antes mencionada exime a la institución de responsabilidad, entonces se debe enfatizar que desde el momento en que el estudiante es aceptado y matriculado en el sistema, todo aquello que suceda respecto a su formación será responsabilidad de la institución hasta que logre obtener su título bajo estándares de calidad establecidos al inicio de su incorporación. Por otro lado, algunos autores hacen distinción entre la deserción y la suspensión de estudios, indicando que los estudiantes que reciben apoyo financiero tienen una alta probabilidad de inscripción y los estudiantes que reciben apoyo académico el primer año tienen una alta probabilidad de continuar sus estudios (Strattom et al., 2008). El concepto que el equipo investigador establece que la deserción es el retiro del programa de estudios sin tomar en cuenta la posible reincorporación debido a que, por lo general, esto no supera el $2 \%$ de la cohorte en estudio, por lo tanto, aquello no modifica significativamente la medición. Además, el seguimiento se realiza año a año y siempre se calcula la deserción respecto del año de ingreso de la cohorte.

\section{Permanencia}

\section{En la ecuación (1), tenemos que}

$n$ : es el Número de años correspondiente al programa de estudios; $E_{(x)}^{c}$ : es el Número de Estudiantes el año "x "de la cohorte "c"; $N_{(c)}$ : es el Número total de Estudiantes correspondiente al año de la cohorte "c"; $T_{(c)}$ : es el Número titulados el año "x" correspondiente a la cohorte "c" 'para $x>n$, si $x<n T_{(c)}=0$; y $P_{(x)}^{c}$ : es el Porcentaje de estudiantes de la cohorte "c " que permanecen el año "x".

$$
P_{(x)}^{c}=100 \frac{E_{(x)}^{c}+T_{(c)}}{N_{(c)}}
$$

La figura 1, describe el concepto de permanencia donde la permanencia teórica es aquella que todos sus ingresantes se titulan el año indicado para la carrera, y la permanencia real, donde se indica un área que es definida como el IGD o Índice Geométrico de Deserción, nótese que en la ecuación 1 se indica que la permanencia está definida por los estudiantes de la cohorte más los titulados, con la finalidad de medir correctamente la deserción, después del año de término de la carrera la caída de la cohorte se debe a la deserción y la titulación. 


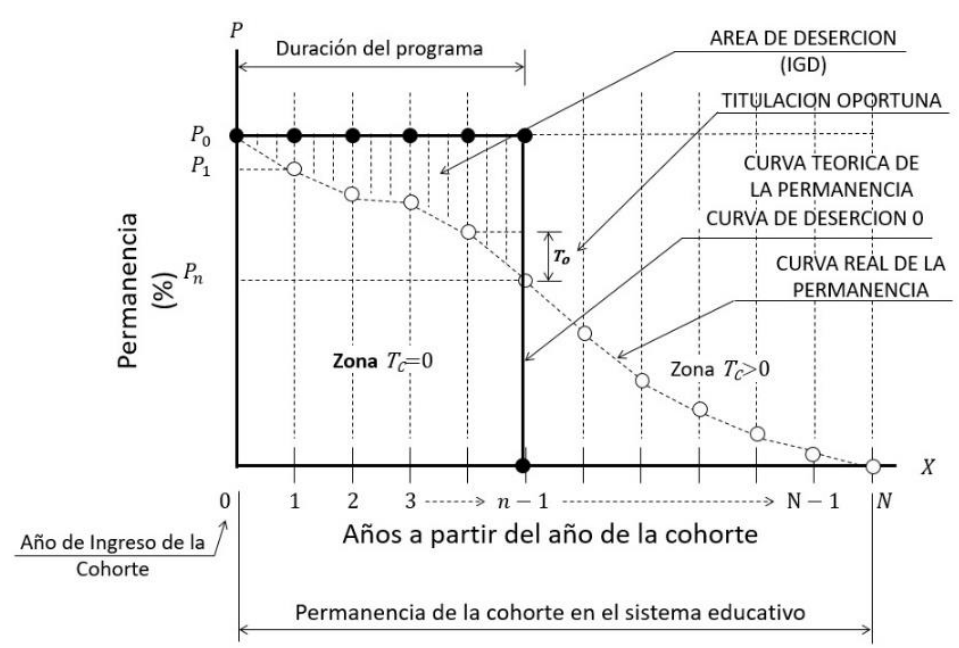

Fig. 1: Esquema de la Permanencia estudiantil universitaria.

\section{Índice de Deserción}

La investigación propone un nuevo indicador para observar la deserción de los programas de estudio, donde se define la permanencia como la cantidad de estudiantes de una cohorte respecto del total de estudiantes del año de ingreso. De esta forma la permanencia teóricamente deberá mantenerse igual durante los años que dure el programa, suponiendo que no existe deserción. Sin embargo, esa condición es excepcional, la realidad indica que la deserción se presenta con intensidad en el primer año por lo que la permanencia deberá disminuir drásticamente el primer año y en menor grado los siguientes. El índice geométrico de deserción (IGD) se define como el área entre la permanencia inicial $\left(P_{0}\right)$ y la permanencia real (ver ecuación 2). Nótese que si no existiera deserción y todos los estudiantes se titulan al termino de los años indicados en su programa de estudio, el IGD es 0 . En esta ecuación, IGD : Índice geométrico de Deserción; $k$ : k-ésimo año de la cohorte en estudio; $n$ : Número de años del programa; y $P_{k}$ : Permanencia del k-ésimo año.

$$
I G D_{n}=\frac{(2 n-1) P_{0}-P_{n}}{2}-\sum_{k=1}^{n} P_{k}
$$

Por ejemplo, para una carrera de cinco años, el indicador se mediría por la ecuación 3:

$$
I G D_{5}=\frac{9 P_{0}-P_{5}}{2}-\left(P_{1}+P_{2}+P_{3}+P_{4}\right)
$$

Para el caso de estudio de la presente investigación se utilizaron datos de registro curricular en el periodo 2012-2017, de un departamento del ámbito de las tecnologías industriales, con cuatro carreras de 3 años de duración con 555 estudiantes en el año 2012, donde 152 corresponden a la carrera C1 (Automatización), 184 a la C2 (Construcción), 136 a la C3 (Telecomunicaciones) y 83 a la C4 (Mantenimiento) (ver Tabla 1). Para todos los efectos se hace referencia a las carreras como $C_{1}, C_{2}, C_{3}, C_{4}$ y al departamento como D. Para tal efecto se utilizó la ecuación 4. La misma que corresponde a una carrera de 3 años de duración.

$$
I G D_{3}=\frac{5 P_{0}-P_{3}}{2}-\left(P_{1}+P_{2}\right)
$$

\section{RESULTADOS Y ANALISIS}

Los resultados se exponen dividiendo el análisis en dos dimensiones, primero se muestra la visión general donde se observan la Permanencia por departamento y por carrera, finalmente se muestra la visión de género que muestra la variable Permanencia y Deserción por género.

\section{Visión General}

Se presenta a continuación un análisis de los resultados visto de forma general, solo distinguiendo las dimensiones por carreras, lo que mostrará cuanto aportan las carreras a los cambios del departamento. En la tabla 1, se muestran los resultados para la cohorte 2012 de las cuatro carreras en el periodo 2012-2017, donde el año de titulación para esta cohorte es el 2014. Los datos se encuentran clasificados por género y 
por carrera, mostrando el cálculo de la permanencia por género y total. La figura 2, muestra la permanencia de la carrera C1 para la cohorte 2012, 2013, 2014 y 2015 para los seis primeros años a partir del 2012. Se puede ver la tendencia similar de las cohortes donde las cohortes permanecen en la institución hasta por 6 años en carreras que solo duran tres años, esta tendencia es sin duda negativa y se deberán implementar acciones que reviertan esta tendencia. La figura 3, muestra la permanencia de la carrera C2 para la cohorte 2012, 2013, 2014 y 2015. Se puede ver la tendencia de la cohorte 2012, y cambios positivos en la tendencia en las cohortes 2013 y 2014. Las únicas cohortes que tienen un comportamiento cuasi teórico son las cohortes 2012 y 2013, mientras que el resto de carreras tienen una deserción muy marcada después de su tercer año, momento en el que se debió acabar la carrera. Por lo demás, se puede observar la tendencia sin duda negativa de la cohorte 2012 permaneciendo el sistema seis años, mientras la cohorte 2014 tiene una marcada mejoría. La cohorte 2015 sigue la misma tendencia por lo que se proyecta una disminución de la permanencia en el sistema. Estas imágenes de la permanencia de las cohortes permiten observar que la tendencia de la permanencia en el sistema es definitivamente cerca de los seis años. No olvidemos que estas carreras se ofrecen con una duración de tres años.

Tabla 1: Resultados para la cohorte 2012. Donde, F: Número de estudiantes mujeres, M: Número de estudiantes varones, ST: Subtotal de estudiantes, PF: Permanencia femenina (\%), PM: Permanencia masculina (\%), PT: Permanencia total (\%).

\begin{tabular}{|c|c|c|c|c|c|c|c|}
\hline 555 & & 2012 & 2013 & 2014 & 2015 & 2016 & 2017 \\
\hline 152 & $\mathrm{~F}$ & 6 & 6 & 4 & 4 & 3 & 0 \\
\hline \multirow{5}{*}{$C 1$} & $M$ & 33 & 31 & 25 & 24 & 13 & 3 \\
\hline & ST & 39 & 37 & 29 & 28 & 16 & 3 \\
\hline & PF & 3,95 & 3,95 & 2,63 & 2,63 & 1,97 & 0,00 \\
\hline & PM & 21,71 & 20,39 & 16,45 & 15,79 & 8,55 & 1,97 \\
\hline & PT & 25,66 & 24,34 & 19,08 & 18,42 & 10,53 & 1,97 \\
\hline 184 & $\mathrm{~F}$ & 11 & 11 & 10 & 10 & 6 & 1 \\
\hline \multirow{5}{*}{$C 2$} & $M$ & 47 & 46 & 43 & 36 & 18 & 6 \\
\hline & ST & 58 & 57 & 53 & 46 & 24 & 7 \\
\hline & $\mathrm{PF}$ & 5,98 & 5,98 & 5,43 & 5,43 & 3,26 & 0,54 \\
\hline & $P M$ & 25,54 & 25,00 & 23,37 & 19,57 & 9,78 & 3,26 \\
\hline & $\mathrm{PT}$ & 31,52 & 30,98 & 28,80 & 25,00 & 13,04 & 3,80 \\
\hline 136 & $\mathrm{~F}$ & 2 & 2 & 2 & 2 & 1 & 0 \\
\hline \multirow{5}{*}{ C3 } & $M$ & 27 & 24 & 16 & 17 & 8 & 3 \\
\hline & ST & 29 & 26 & 18 & 19 & 9 & 3 \\
\hline & $\mathrm{PF}$ & 1,47 & 1,47 & 1,47 & 1,47 & 0,74 & 0,00 \\
\hline & $P M$ & 19,85 & 17,65 & 11,76 & 12,50 & 5,88 & 2,21 \\
\hline & PT & 21,32 & 19,12 & 13,24 & 13,97 & 6,62 & 2,21 \\
\hline 83 & $\mathrm{~F}$ & 2 & 2 & 2 & 2 & 1 & 0 \\
\hline \multirow{5}{*}{$C 4$} & $M$ & 11 & 9 & 7 & 6 & 5 & 2 \\
\hline & ST & 13 & 11 & 9 & 8 & 6 & 2 \\
\hline & PF & 2,41 & 2,41 & 2,41 & 2,41 & 1,20 & 0,00 \\
\hline & PM & 13,25 & 10,84 & 8,43 & 7,23 & 6,02 & 2,41 \\
\hline & PT & 15,66 & 13,25 & 10,84 & 9,64 & 7,23 & 2,41 \\
\hline 555 & $F$ & 21 & 21 & 18 & 18 & 11 & 1 \\
\hline \multirow{5}{*}{$D$} & $M$ & 118 & 110 & 91 & 83 & 44 & 14 \\
\hline & ST & 139 & 131 & 109 & 101 & 55 & 15 \\
\hline & PF & 3,78 & 3,78 & 3,24 & 3,24 & 1,98 & 0,18 \\
\hline & $P M$ & 21,26 & 19,82 & 16,40 & 14,95 & 7,93 & 2,52 \\
\hline & PT & 25,05 & 23,60 & 19,64 & 18,20 & 9,91 & 2,70 \\
\hline
\end{tabular}

La figura 4, muestra la permanencia de la carrera C3 para la cohorte 2012, 2013, 2014 y 2015. Donde se observa la misma tendencia que la carrera C1. Nótese que la cohorte 2012 permanece en el sistema por más de seis años, siendo las carreras estudiadas de tres años de formación según programa de estudios. Las otras cohortes siguen la misma tendencia y se espera que duren la misma cantidad de años en el sistema. Solo la cohorte 2014 parece disminuir lo que permite estimar que esta corte permanecerá menos años que la 2012. 


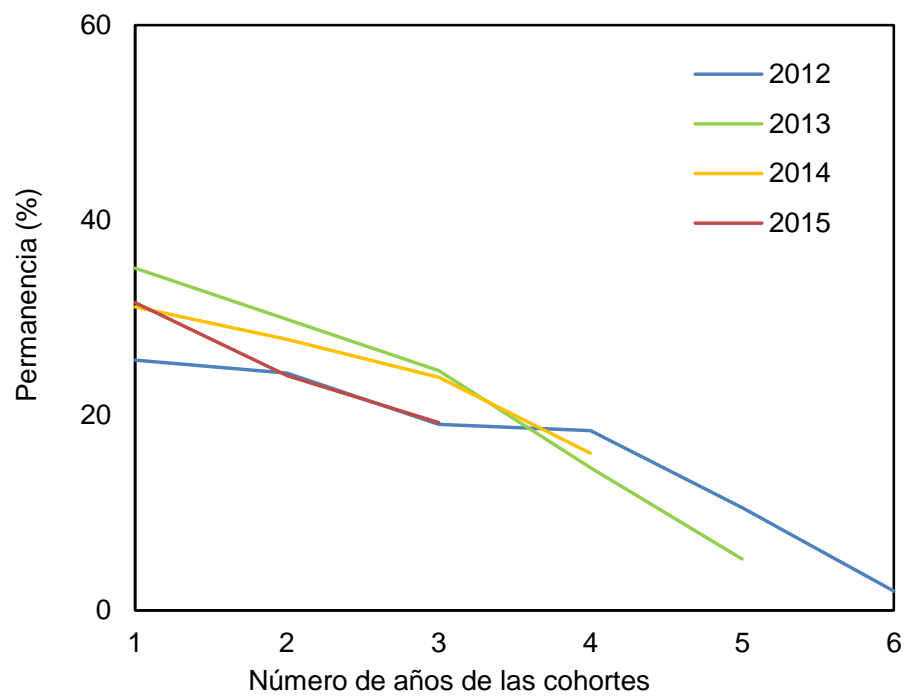

Fig. 2: Permanencia estudiantil para cuatro cohortes para la carrera C1.

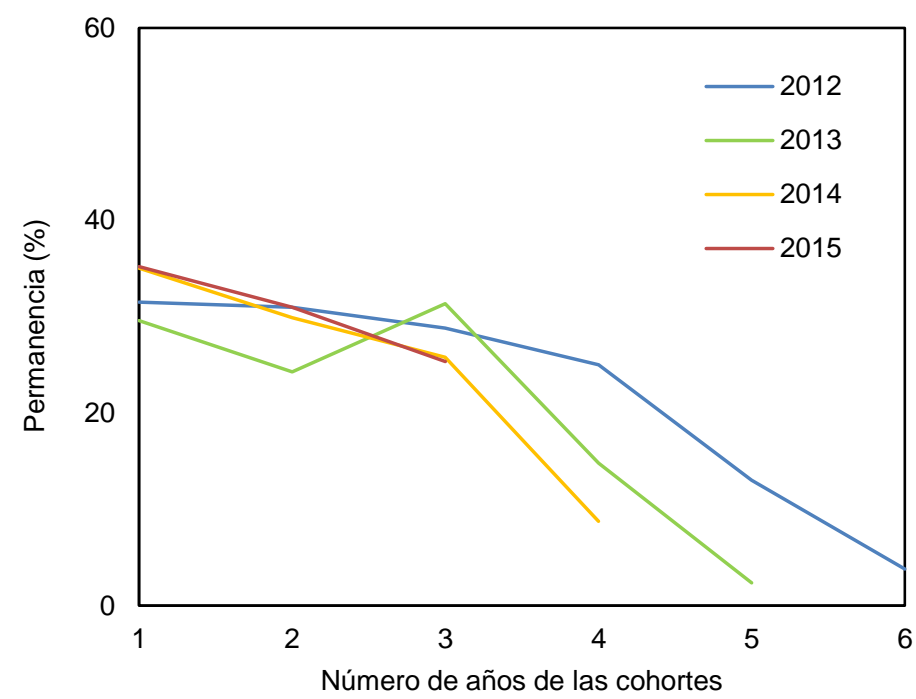

Fig. 3: Permanencia estudiantil para cuatro cohortes parca la carrera C2.

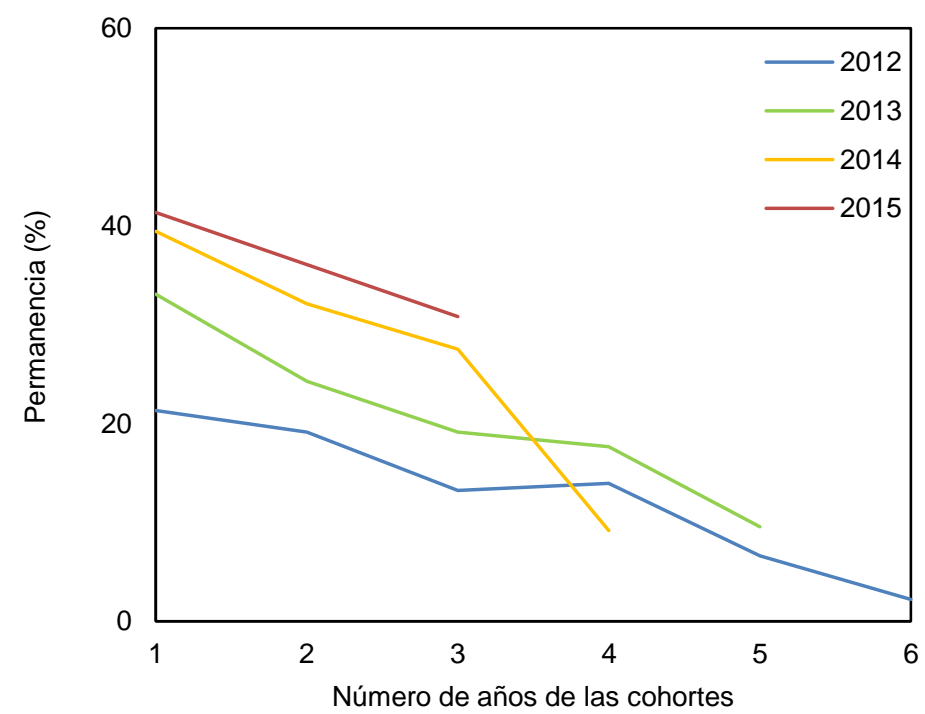

Fig. 4: Permanencia estudiantil para cuatro cohortes parca la carrera C3. 


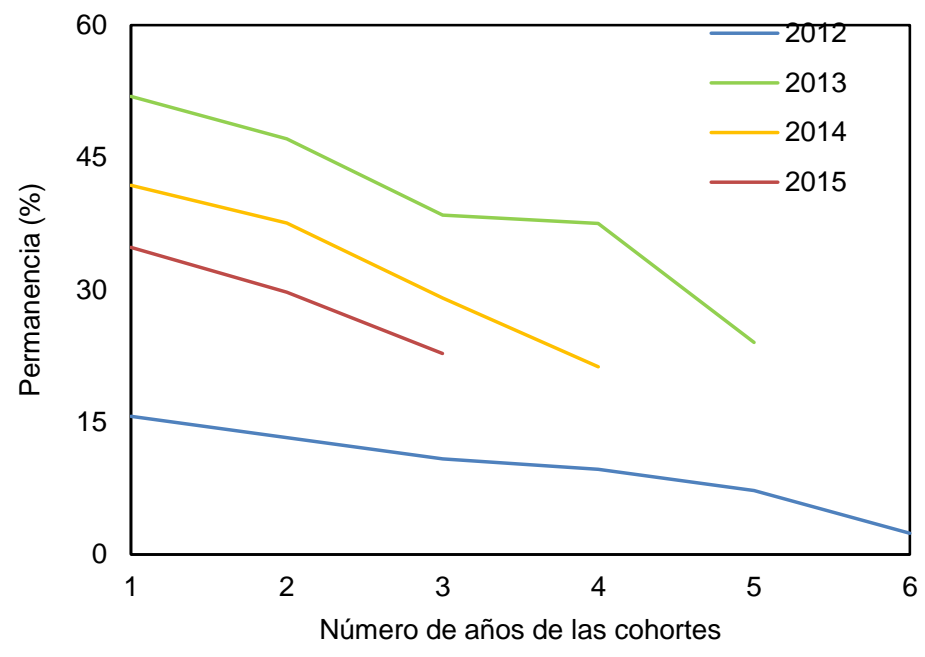

Fig. 5: Permanencia estudiantil para cuatro cohortes para la carrera C4.

Tabla 2: IGD para el periodo 2012-2015. Donde, C1 (\%), C1 (\%), C2 (\%), C3 (\%) y C4 (\%).

\begin{tabular}{|c|c|c|c|c|}
\hline & 2012 & 2013 & 2014 & 2015 \\
\hline$C 1$ & 11,51 & 26,02 & 18,06 & 25,94 \\
\hline$C 2$ & 6,52 & 10,95 & 27,58 & 19,01 \\
\hline$C 3$ & 13,97 & 30,51 & 34,40 & 21,05 \\
\hline$C 4$ & 10,24 & 25,48 & 27,30 & 34,49 \\
\hline$D$ & 10,27 & 35,05 & 31,62 & 35,83 \\
\hline
\end{tabular}

La figura 5, muestra la permanencia de la carrera C4 para la cohorte 2012, 2013, 2014 y 2015. Donde se observa la misma tendencia que la carrera $\mathrm{C} 1$. Después de observar las cuatro figuras, se deprende que las carreras $\mathrm{C} 1, \mathrm{C} 3$ y $\mathrm{C} 4$ siguen el mismo comportamiento de la permanencia con una tendencia a permanecer 6 años, siendo la carrera C2 la única con ciertos indicios de cambio. Este similar comportamiento es de mucha preocupación porque son cuatro cohortes de la misma carrera, esto lleva a la reflexión sobre las razones de tal comportamiento por que surgen dos escenarios, el primero es la matriz docente de la carrera y el segundo la matriz metodológica, los mismos que deberán realizarse a profundidad en próximas investigaciones. La tabla 2, muestra los resultados de los cálculos (según ecuación 4) del IGD para las cohortes 2012, 2013, 2014 y 2015, distribuidas por carrera y consolidadas por departamento. De los datos de la tabla se desprende que el departamento tiene una sostenida deserción, donde esta tiene una tendencia perniciosa de incremento.

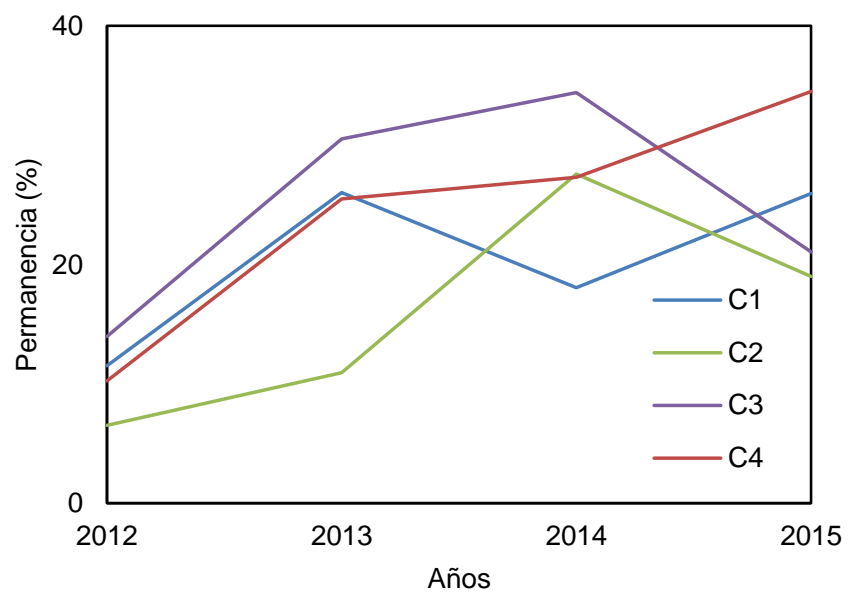

Fig. 6: IGD para cuatro cohortes y las cuatro carreras. 
En la figura 6, se puede observar el comportamiento del IGD por cohorte, notándose un serio crecimiento sostenido que es indeseable en todo sistema educativo. Solo C2 y C3 muestran una leve disminución que no logra establecer un patrón de descenso, faltaría mayor observación, es decir mayor cantidad de años de registro.

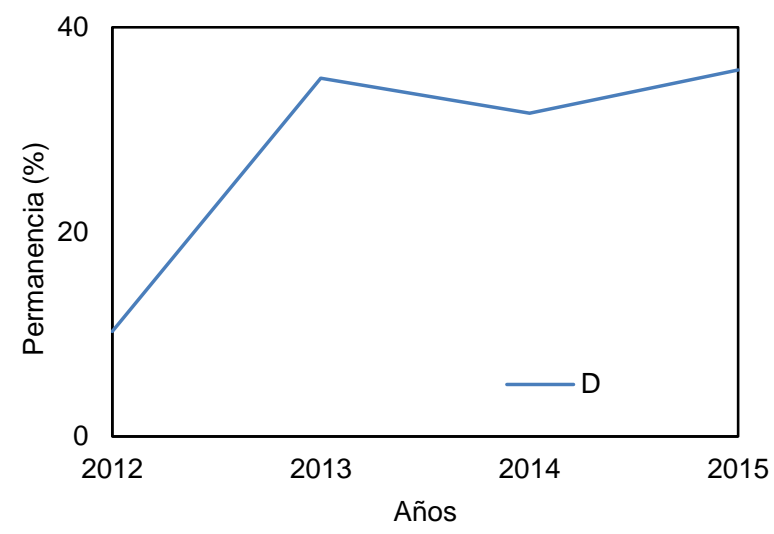

Fig. 7: IGD departamental.

En la Fig. 7, se observa el IGD consolidado para el departamento D, mostrando una tendencia a disminuir después del 2013, sin lograr ser inferior al 2012, y retomando la gradiente positiva. Esta tendencia es negativa para el sistema, pues logra establecer que en cuatro años no se ha podido revertir la tendencia al alza.

Tabla 3: Pendiente equivalente del IGD para las cuatro carreras para el periodo 2012-2015. Donde, C1 (\%), C1 (\%), C2 (\%), C3 (\%) y C4 (\%).

\begin{tabular}{|c|c|}
\hline Carrera & Pendiente IGD \\
\hline C1 & 3,5301 \\
\hline C2 & 5,4108 \\
\hline C3 & 2,5135 \\
\hline C4 & 7,4582 \\
\hline D & 2,3958 \\
\hline
\end{tabular}

La tabla 3, muestra los valores de las pendientes de las tendencias lineales de cada carrera y se observa que la carrera C4 es la de mayor tendencia al alza, contrastando con la pendiente de la carrera C3 que es la más baja. Pero lo preocupante es que el departamento tiene una tendencia positiva, lo que indiaca que cada año desertan más.

\section{Visión de Genero}

La figura 8, muestra los comportamientos del IGD para las estudiantes de las cuatro carreras, no se puede dejar de mencionar la estabilidad del indicador para todas las cohortes demostrando que las estudiantes tienen una alta tolerancia a la frustración no solo mantienen bajo el indicador sino también sin grandes cambios en cada cohorte. Por otro lado, la carrera C1 tiene el más bajo valor del IGD mientras las carreras restantes contrastan, pero sin grandes variaciones. Resalta el comportamiento cuasi cíclico de las carreras C2 y C4, nótese que las cohortes 2013 aumentan en deserción mientras que ambas disminuyen en la cohorte 2014. Este comportamiento es propio no de los estudiantes, aun cuando falta profundizar la investigación las razones están cerca de la matriz docente y su metodología, nuevamente se hace visible que las razones de la deserción se encuentran dentro del sistema y no fuera él. Además, las carreras $\mathrm{C} 1$ y C3 contrastan con sus carreras pares, teniendo un comportamiento bastante estable, destacando la carrera C1 con un comportamiento de deserción bastante baja durante cuatro cohortes.

La figura 9, muestra los comportamientos del IGD para los estudiantes del género masculino, observándose los elevados valores del IGD relativo y su gradiente positiva para las cuatro carreras. La carrera C1 nuevamente se destaca por sus grandes valores del indicador y su mayor gradiente. Lo destacable en esta figura es sin duda el sostenido crecimiento del indicador lo que determina que el departamento tendrá grandes problemas en el futuro y se deberán tomar medidas específicas para modificar el comportamiento de la deserción estudiantil. 

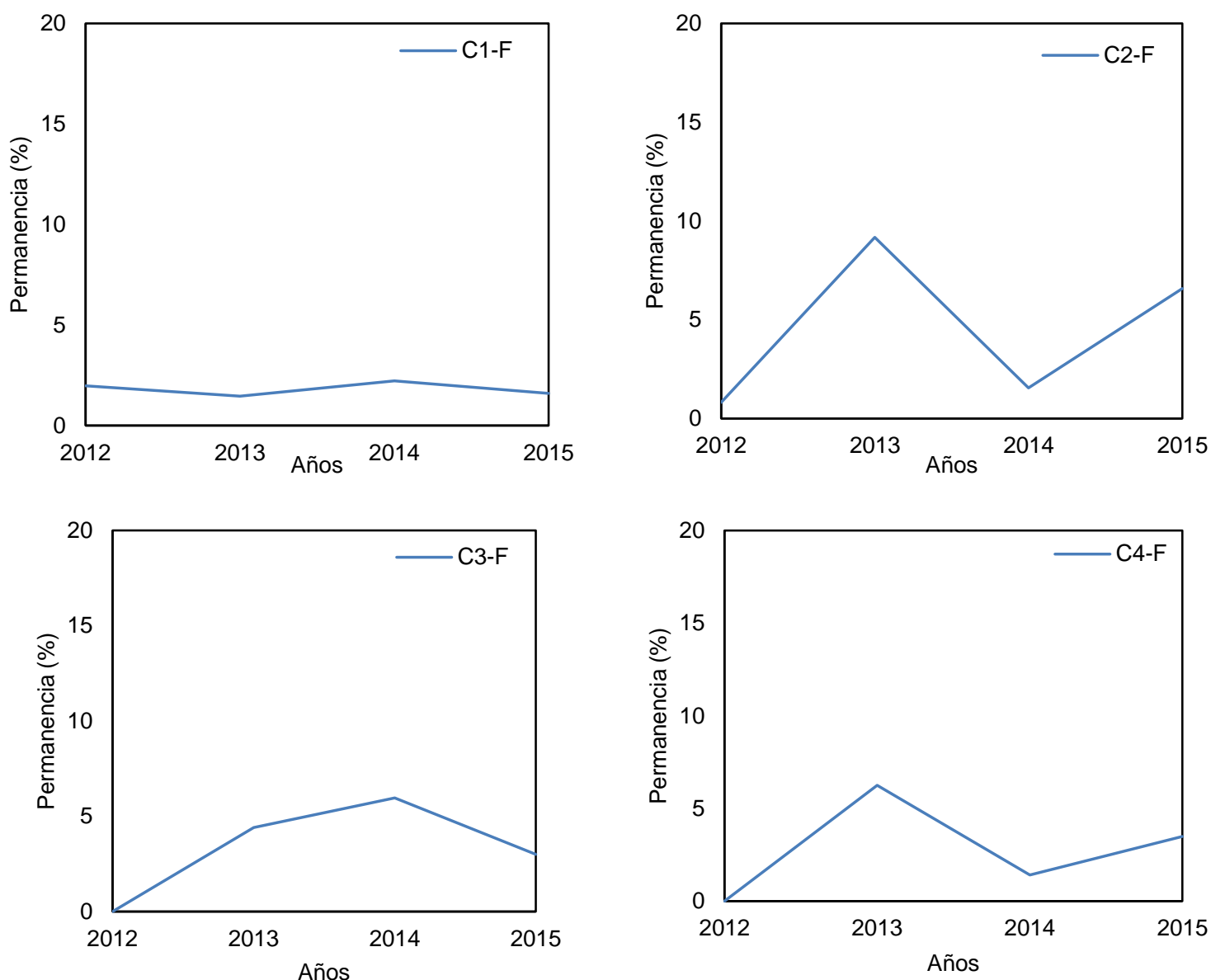

Fig. 8: IGD Femenino por carrera.
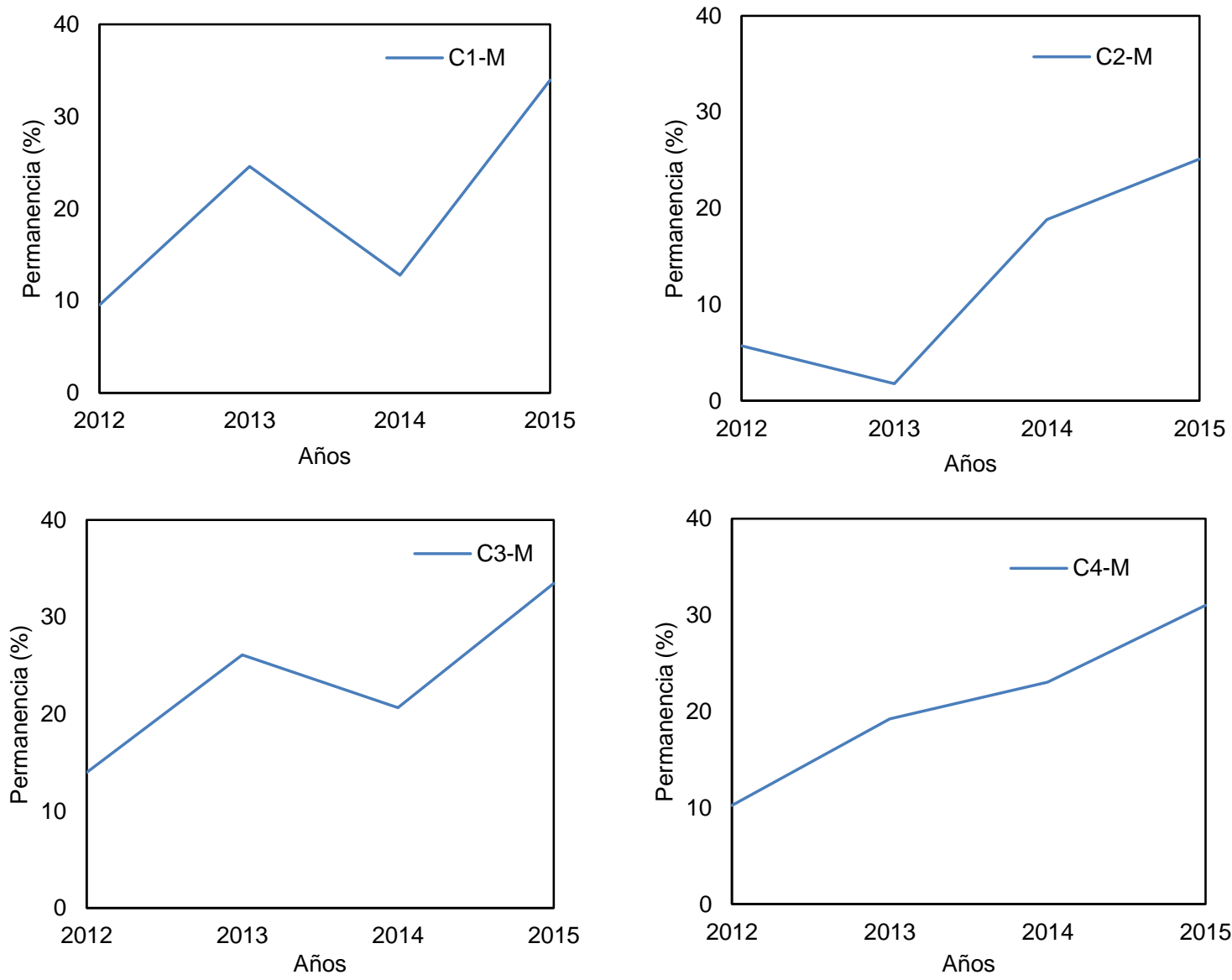

Fig. 9: IGD Masculino por carrera. 
La figura 10, muestra la comparación del IGD departamental para ambos géneros estudiantiles, observándose el contraste de los comportamientos, primero que los IGD femeninos son más bajos que los del género masculino, y segundo que la gradiente del IGD femenino es sensiblemente menor que el masculino y por último la horizontalidad del IGD femenino es destacable frente a la del masculino.
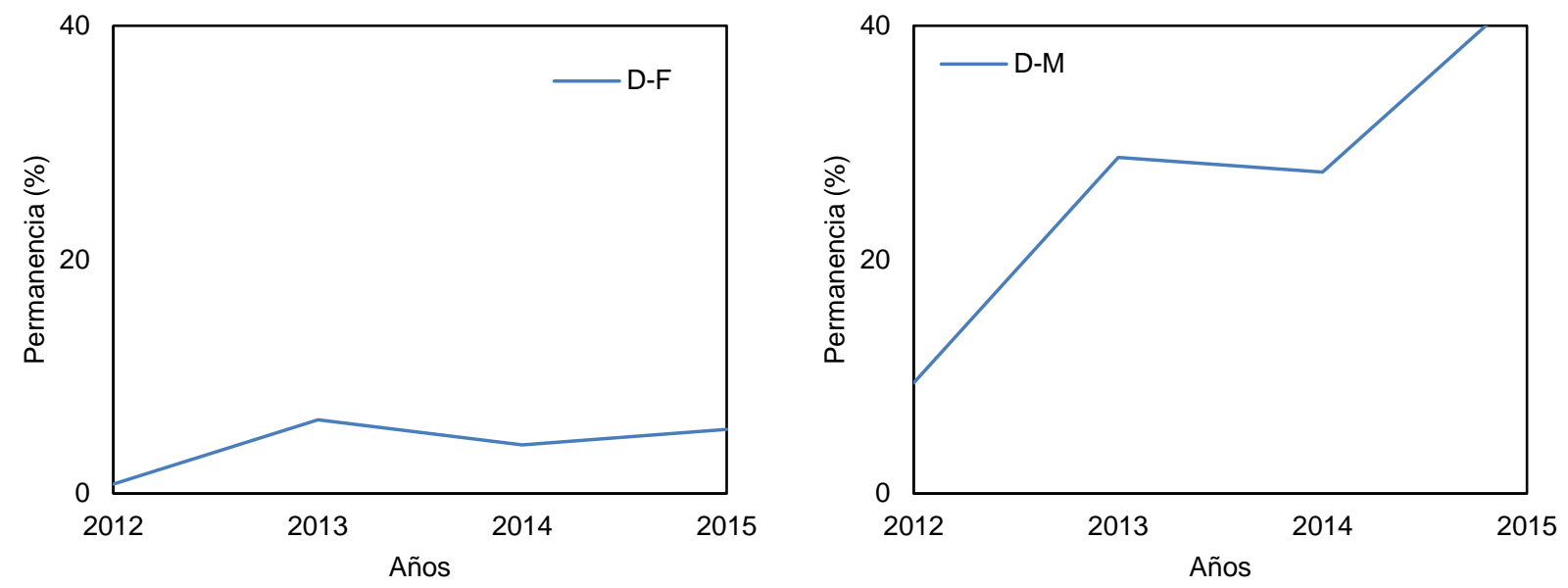

Fig. 10: IGD Departamental por género.

\section{CONCLUSIONES}

El estudio muestra una deserción con clara tendencia al alza. EI IGD muestra resultados que marcan el crecimiento, sin embargo, existen algunas carreras que han empezado a disminuir como es el caso de C2 y C3. El nuevo indicador es muy versátil, debido a que establece la medición basado en la historia de la deserción del plantel, el presente indicador será presentado para su utilización en la unidad. Además, el indicador basado en la superficie complementaria a la permanencia estudiantil logra medir con mayor exactitud la deserción, ayudando a entender con mayor precisión este fenómeno.

Otra observación destacable es el comportamiento del género femenino que se condice con muchos sobre la alta tolerancia a la frustración de este género, frente a los elevados valores del IGD que se observan en el género masculino. Es importante realizar seguimiento específico a las cohortes ingresantes en las instituciones de educación superior para transparentar los procesos de deserción que el sistema genera. El presente indicador es una nueva forma de medir la deserción y permite observar el comportamiento de las cohortes durante todo el proceso educativo, desde el ingreso de la cohorte hasta el retiro del último integrante, de esta forma se logra observar la acción del sistema sobre los estudiantes y poder dar uso a este indicador como elemento predictor y registrador de la deserción actual y futura de la institución.

\section{AGRADECIMIENTOS}

Para el Proyecto Fortalecimiento USACH USA1398_GM181622 de la Universidad de Santiago de Chile USACH, Proyecto IMP-ING-2660 de la Vicerrectoría de Investigaciones de la Universidad Militar Nueva Granada y al Grupo de Investigación en Nuevas Tecnologías (GINT-DTI-USACH) por el importante apoyo al desarrollo de la investigación.

\section{REFERENCIAS}

Alegre de la Rosa, O.M.y L.M. Villar, Indicadores y control estadístico para el seguimiento y evaluación de preferencias de aprendizaje de estudiantes universitarios, Revista de Educación a Distancia, 55, Art. 2 (2017)

Baeza-Rivera, M.J., A. Antivilo y L. Rehbein, Diseño y Validación de una Escala de Preparatividad Académica para la Educación Superior en Chile, Formación Universitaria, 9(4), 63-74 (2016)

Durkeim, E., Historia de la educación y de las doctrinas pedagógicas, La evolución pedagógica en Francia, Madrid, Ediciones La Piqueta (1992)

Esteban, M., A. Bernardo, E. Tuero, A. Cervero y J. Casanova, Variables influyentes en progreso académico y permanencia en la Universidad, European Journal of Education and Psychology, 10(1), 75-81 (2017)

Gregori, P., V. Martínez y J. J. Moyano-Fernández, Basic actions to reduce dropout rates in distance learning, Evaluation and Program Planning, 66(1), 48-52 (2018) 
Grosset, J.M., Patterns of integration, commitment, and student characteristics, and retention among younger and older students, Research in Higher Education, 32 (2), 159-178 (1991)

Himmel, E., Modelos de Análisis de la Deserción Estudiantil en la Educación Superior, Calidad en la Educación, 1(1), 91-107 (2002)

McFarland, J., B. Hussar y otros nueve autores, The Condition of Education 2017. NCES 2017-144, National Center for Education Statistics (ED), American Institutes for Research (AIR), (2017)

Miranda, M.A. y J. Guzmán, Análisis de la Deserción de Estudiantes Universitarios usando Técnicas de Minería de Datos, Formación Universitaria, 10(3), 61-68 (2017)

Montes-Gutiérrez, I., P. Almonacid-Hurtado y otros tres autores, Análisis de La Deserción Estudiantil en Los Programa de Pregrado de la Universidad EAFIT, Cuadernos de Investigación, Universidad EAFIT, Abril (2010)

Pascarella, E.T. y P.T. Terenzini, Predicting freshmen persistence and voluntary dropouts decisions from a theoretical model, Journal of Higher Education, 51(1), 60-75 (1980)

Rodríguez, A., Methodological Intervention in Class: Learning From Comprehension, Edulearn15 Proceedings, 1(1), 1008-1012 (2015)

Rodríguez, A., Academic Performance and Learning Style by Kolb of Students in Drop Out, Iceri2010 Proceedings, 1(1), 48-56 (2010)

Spady, W.G., Dropouts from higher education: An interdisciplinary review and synthesis, Interchange, 1 (1), $64-85$ (1970)

Suhlmann, M., K. Sassenberg, B. Nagengast y U. Trautwein, Belonging mediates effects of studentuniversity fit on wellbeing, motivation, and drop-out intention, https://doi.org/10.1027/1864-9335/a000325, Social Psychology, 49, 16-28 (2018)

Tinto, V., Definir la deserción: Una Cuestión de Perspectiva, (trad.), Revista de la Educación Superior, 18(71), 1-9 (1989) 
